\title{
THE CIRCUMSTELLAR MATTER OF CYGNUS X-3
}

\author{
S. KITAMOTO AND K. KAWASHIMA
}

Department of Earth and Space Science, Graduate School of Science, Osaka University, 1-1, Machikaneyama-cho, Toyonaka, Osaka, 560, JAPAN

\section{Introduction}

$\mathrm{X}$-ray source in Cyg X-3 is embedded in the photo-ionized circumstellar gas. Recombination edges and emission lines were detected by ASCA observation (Kawashima, Kitamoto 1996; Liedahl, Paerels 1996). The strength of the $\mathrm{K} \alpha$ line and the recombination edge are simply determined by the atomic process. Therefore, the comparison of observed these intensities is a good tool to study the Cyg X-3 system.

\section{Analysis and Results}

The emission measure can be defined as $\int n_{e} n_{z}^{i+1} d V \mathrm{~cm}^{-3}$. The line intensity is a function of a product of the emission measure and of the emissivity, which is a function of the electron temperature. Therefore observed line intensity makes a constraint on a map of the emission measure and electron temperature. The recombination edge intensity also gives a constraint on the map. Since the energy of the emission line is smaller than that of the recombination edge, absorption by the circum- and inter-stellar matter should be taken into account. However, the absorption measure has not well established. Here, we applied the absorption measure of $1.5 \times 10^{22} \mathrm{H}$ atoms $\mathrm{cm}^{-2}$; this value is rather small value comparing previously reported values (e,g, Kitamoto et al. 1987). Figure 1 shows the derived constraint map for the $\mathrm{H}$-like $\mathrm{S}$. There is no overlapped region.

\section{Discussion}

Before we conclude the above result, we have to consider the effect of the absorption edge. The absorption edge has the same energy as the recom- 


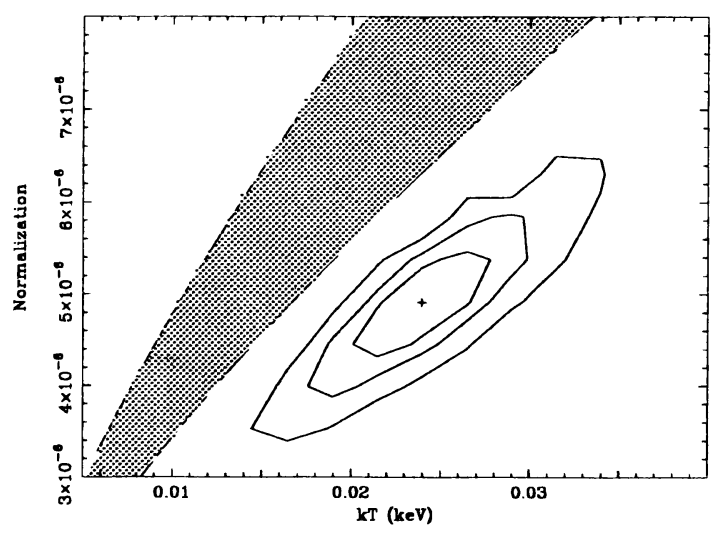

Figure 1. Comparison between the line intensity and the recombination edge intensity of $\mathrm{H}$-like $\mathrm{S}$. Contours are $68 \%, 90 \%$ and $99 \%$ confidence region derived from the recombination edge on the (Electron Temperature v.s. Emission Measure) space. Normalization means the emission measure defined in text, in an unit of $\frac{10^{-14}}{4 \pi D^{2}}$. Gray region is a $99 \%$ confidence region derived from the line intensity.

bination edge has. Therefore, the absorption edge feature could affect the intensity of the recombination edge. If we assume the spherically symmetric and ionization equilibrium plasma, the absorption optical depth can be estimated from the column density of the ions, which we can derive from the recombination edge. The obtained absorption optical depth at the threshold energy is less than 0.01 and the resultant effect on the intensity of the recombination edge is smaller than the statistical uncertainty. Consequently, the effect of the photoelectric absorption edge structure can be neglected in our case.

The most plausible reason of the observed strong line is an anisotropic configuration. Since the main lines that we are considering are resonance lines, which have a large scattering cross section. If geometrical obscuration occurs, for example, the line of sight to the continuum source is blocked, the ratio of the scattered photons to the recombination edge will become large.

\section{References}

Kawashima K., Kitamoto S. 1996, PASJ, 48, L113

Liedahl D. A., Paerels F. 1996, ApJ, 468, L33

Kitamoto S, Miyamoto S., Matsui W., Inoue H., 1987, PASJ, 39, 259 